\title{
Insulated Loop Antenna Immersed in a Conducting Medium
}

\author{
James R. Wait
}

\begin{abstract}
A solution is given for the fields of a circular loop in a conducting medium. The loop is assumed to have a uniform current, and it is enclosed by a spherical insulating cavity. The impedance of the loop is also considered. It is shown that the power radiated from the loop varies approximately as the reciprocal of the radius of the cavity for a specified loop current. Furthermore, if the cavity is electrically small, relative to the external medium, the radiation field is not significantly affected by the presence of the cavity.
\end{abstract}

\section{Introduction}

There has been some interest shown in recent years on the subject of radiating systems immersed in dissipative media $[1$ to 9$] .{ }^{1}$ Such a condition exists when transmitting antennas are located in sea water, buried beneath the ground, or surrounded by an ionized medium, such as the ionosphere. Most investigators have considered only the propagation aspects of the general problem. That is, attention was devoted only to the radiated field for a specified antenna current. An associated, and a usually much more difficult problem; is the calculation of the impedance of the antenna.

Tai [1], in an interesting analysis, showed that for a Hertzian electric dipole in a dissipative medium, infinite power is required to produce a finite field strength at some distant point in the medium. This difficulty is overcome when the dipole is placed in an insulated cavity. It has also been shown that a magnetic-dipole-type antenna was more efficient than an electricdipole-type antenna $[2,5]$.

It is the purpose of the present paper to reinvestigate the problem treated before. Specifically, the impedance and fields are calculated for a circular loop with a spherical insulating cavity, all immersed in a homogeneous dissipative medium. In the previous analysis [5], the loop was represented as a magnetic dipole at the center of the cavity. It was implied that the dimensions of the loop were small compared to those of the cavity. In this sequel, these limitations are removed by treating the problem from another viewpoint.

In terms of spherical coordinates $(r, \theta, \phi)$ the insulating cavity is defined by $r<a$ and has a dielectric constant, $\epsilon_{0}$. The exterior homogeneous dissipative medium, $r>a$, has a dielectric constant, $\epsilon$, and a conductivity, $\sigma$. The permeability, $\mu$, of both regions is taken to be that of free space. The loop, for the moment, is considered to be a single turn carrying a uniform current, $I$. The coordinates of the loop are $\theta=\beta$ and $r=b$, so that the axis of the (circular) loop passes through the center of the spherical cavity (see fig. 1).

The dimensions of the cavity are taken to be very small compared to the wavelength, which suggests that a quasi-static approach to the problem should be adopted. As a consequence, the fields are a solution of Laplace's equation inside the cavity and a solution of the wave equation outside. Although it is not necessary to invoke the quasi-static assumption for the field calculation, it leads to the desired result in a direct fashion without becoming encumbered with a myriad of spherical Bessel functions. Furthermore, in any instance where the cavity was not small compared to the wavelength, the assumption of uniform current around the loop would be a poor one for a localized generator.

\footnotetext{
1 Figures in brackets indicate the literature references at the end of this paper.
} 


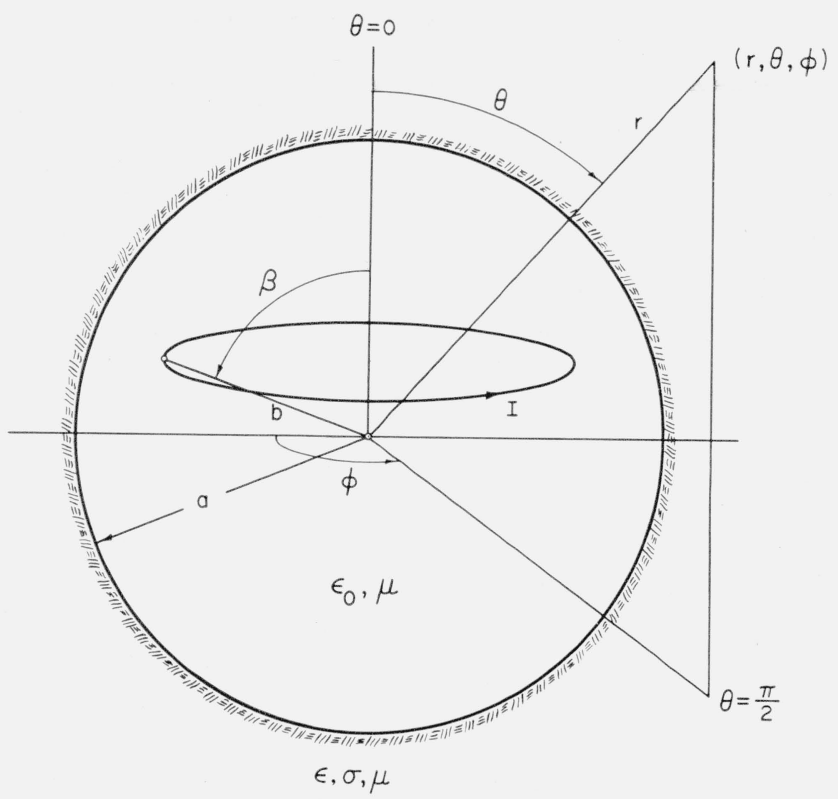

Figure 1. Circular loop antenna in a spherical insulating cavity.

The surrounding homogeneous dissipative medium is infinite in extent.

\section{Formal Solution}

In view of the polar symmetry of the stated problem, the usual vector potential, $\vec{A}$, has only an azimuthal component, $A$. The nonvanishing field components are then given by

$$
\begin{aligned}
E_{\phi} & =-i \mu \omega A, \\
H_{\theta} & =-\frac{1}{r} \frac{\partial(A r)}{\partial r} \\
H_{r} & =\frac{1}{r \sin \theta} \frac{\partial}{\partial \theta}(A \sin \theta) .
\end{aligned}
$$

The (quasi-static) form of the vector potential of the primary field of a circular loop in an insulating space can be expressed in terms of spherical harmonics (see appendix):

$$
r A^{p}=\frac{I \sin \beta}{2} \sum_{n=1}^{\infty} \frac{b}{n(n+1)}\left(\frac{b}{r}\right)^{n} P_{n}^{1}(\cos \theta) P_{n}^{1}(\cos \beta)
$$

for $r>b$, where $P_{n}^{1}$ is the associated Legendre function. To account for the spherical wall of the cavity, a secondary field, $A^{s}$, must be added. Because it is also a solution of Laplace's equation and is finite $r=0$, it must be of the form

$$
r A^{s}=\frac{I \sin \beta}{2} \sum_{n=1}^{\infty} \frac{S_{n} b}{n(n+1)}\left(\frac{r}{b}\right)^{n+1} P_{n}^{1}(\cos \theta) P_{n}^{1}(\cos \beta)
$$

for $r<a$, where $S_{n}$ is an unknown coefficient.

The field in the external region is a solution of the wave equation, and furthermore the solution must give rise to outgoing waves at infinity. Therefore,

$$
r A=\frac{I \sin \beta}{2} \sum_{n=1}^{\infty} \frac{T_{n} b}{n(n+1)} \frac{k_{n}(\gamma r)}{k_{n}(\gamma a)} P_{n}^{1}(\cos \theta) P_{n}^{1}(\operatorname{crs} \beta)
$$


for $r>a$, where $k_{n}(z)$ is a spherical Hankel function defined by

$$
k_{n}(z)=e^{-z} \sum_{m=0}^{n} \frac{(n+m) !}{m !(n-m) !(2 z)^{m}},
$$

$\gamma=\left(i \mu \sigma \omega-\epsilon \mu \omega^{2}\right)^{1 / 2}$, and $T_{n}$ is an unknown coefficient. The boundary condition at $r=a$ requires the continuity of $E_{\phi}$ and $H_{\theta}$. The solution of the resulting algebraic equations leads to

and

$$
S_{n}=\left[\frac{n+\alpha_{n}}{(n+1)-\alpha_{n}}\right]\left(\frac{b}{a}\right)^{2 n+1}
$$

where

$$
T_{n}=\left[\frac{(2 n+1)}{(n+1)-\alpha_{n}}\right]\left(\frac{b}{a}\right)^{n}
$$

$$
\alpha_{n}=\left[z \frac{d}{d z} \log k_{n}(z)\right]_{z=\gamma a}
$$

When $S_{n}$ and $T_{n}$ are inserted into eq (3) and (4), the complete solution is obtained.

\section{Discussion of Result}

It is now of interest to investigate the influence of the cavity on the external fields. With this in mind, $A$, for $r>a$, is written in the form

$$
r A=\frac{I \sin \beta}{2} \sum_{n=1}^{\infty} \frac{b}{n(n+1)} \frac{k_{n}(\gamma r) n !}{(2 n) !}(2 \gamma b)^{n} G_{n} \times P_{n}^{1}(\cos \theta) P_{n}^{1}(\cos \beta),
$$

where

$$
G_{n}=\left[\frac{2 n+1}{(n+1)-\alpha_{n}}\right] \frac{(2 n) !}{n !(2 \gamma a)^{n} k_{n}(\gamma a)} .
$$

It can be readily verified that $\lim _{\gamma a \rightarrow 0} G_{n}=1$. When this limiting form is inserted into eq (8), the resulting expression would be the field of a small loop in an infinite dissipative medium without any cavity. Specific values of $G_{n}$ for finite $\gamma a$ are

$$
G_{1}=\frac{3 e^{z}}{3+3 z+z^{2}}
$$

and

$$
G_{2}=\frac{15 e^{z}}{15+15 z+6 z^{2}+z^{3}},
$$

where $z=\gamma a$. The value $G_{1}$ can be regarded as the ratio of the effective dipole moment for a loop in an insulated cavity to that of the loop without any cavity. It agrees with a result given previously [5] by a somewhat different method. $G_{2}$ is the corresponding ratio for the next higher multipole term. For most cases of practical interest, $\gamma a$ is somewhat less than unity, and the multipole terms are not important in the external region when $\gamma r$ is large compared to one. Furthermore, in this case

$$
G_{1}=1+\frac{z^{2}}{6}+\ldots \simeq 1
$$

and the external fields for a specified loop current are not dependent on the presence of the cavity. 
Attention is now turned to the effect of the cavity on the impedance of the circular loop. The impedance of the loop in free space is denoted $Z_{0}$, which is assumed known. The impedance of the loop inside the cavity is then written

$$
Z=Z_{0}+\Delta Z
$$

where $\Delta Z$ is the incremental change due to the finite size of the cavity. $\Delta Z$ is the secondary electric field $E_{\phi}^{s}$ integrated along the circumference for a unit loop current. Therefore,

$$
\begin{gathered}
\Delta Z=i \mu \omega A^{s} \frac{2 \pi b}{I} \\
=i \mu \omega \sin \beta(\pi b) \sum_{n=1}^{\infty} \frac{T_{n}}{n(n+1)}\left(\frac{b}{a}\right)^{2 n+1} P_{n}^{1}(\cos \beta) P_{n}^{1}(\cos \theta),
\end{gathered}
$$

where $T_{n}$ is given by eq $(7)$. The above expression is simplified somewhat in the important case where $|\gamma a|$ is small compared to unity, because then

$$
T_{n} \cong-\frac{2}{(2 n+1)(2 n-1)}(\gamma a)^{2}
$$

neglecting terms containing $(\gamma a)^{3},(\gamma a)^{4}$, etc. Furthermore, if $\theta=\beta=\pi / 2$, it follows that

$$
\begin{aligned}
\Delta Z & \simeq \frac{(\mu \omega)^{2}(\sigma+i \omega \epsilon) S^{2}}{6 \pi a} \sum_{n=1}^{\infty} \frac{6}{n(n+1)(2 n+1)(2 n-1)}\left(\frac{b}{a}\right)^{2 n-2} \\
& \simeq \frac{(\mu \omega)^{2}(\sigma+i \omega \epsilon) S^{2}}{6 \pi a}\left[1+\frac{1}{15}\left(\frac{b}{a}\right)^{2}+\frac{1}{70}\left(\frac{b}{a}\right)^{4}+\ldots\right]
\end{aligned}
$$

where $S=\pi b^{2}$. When displacement currents are negligible (i. e., $\left.\omega \epsilon \ll \sigma\right)$ and $(b / a)^{2} \ll 1$,

$$
\Delta Z \cong \frac{(\mu \omega)^{2} \sigma S^{2}}{6 \pi a} .
$$

For a specified current, $I$, on the loop, the power supplied to the surrounding conducting medium is then given by

$$
P \cong \frac{(\mu \omega)^{2} \sigma I^{2} S^{2}}{12 \pi a}
$$

which agrees with a previous result [5] derived from energy considerations.

Although the preceding analysis refers to a single-turn loop, the results are directly applicable to a loop of $N$ turns by replacing $S$ by $S N$. The appropriate expression must also be used for $Z_{0}$, of course.

It would appear that power radiated from the loop for a specified current varies approximately as the reciprocal of the diameter of the cavity. This conclusion is not modified to any extent by the finite size of the loop in the cavity.

\section{Appendix}

It is well known that the vector potential can be obtained from a volume integration over the contained current. Furthermore, because the loop has a filamental uniform current with a component only in the $\phi$ direction, the vector potential has polar symmetry and has only a $\phi$ component $A$; it is given by the line integral

$$
A^{p}=\frac{I}{4 \pi} \int_{0}^{2 \pi} \frac{\cos \phi_{1}}{R} b \sin \beta d \phi_{1},
$$


where $\phi_{1}$ is the azimuthal coordinate for a point on the loop relative to the reference plane, and where

$$
R^{2}=r^{2}+b^{2}-2 r b \cos \Omega
$$

and $\cos \Omega=\cos \theta \cos \beta+\sin \theta \sin \beta \cos \phi_{1}$. Now the factor $1 / R$ is a solution of Laplace's equation and can be written in terms of spherical harmonics, as is well known [10].

$$
\frac{1}{R}=\sum_{n=0}^{\infty} \sum_{m=0}^{n} \epsilon_{m} \frac{(n-m) !}{(n+m) !} P_{n}^{m}(\cos \theta) P_{n}^{m}(\cos \beta) \cdot \cos m \phi_{1} \begin{cases}b^{n} / r^{n+1} ; & r>b \\ r^{n} / b^{n+1} ; & r<b,\end{cases}
$$

where $\epsilon_{0}=1, \epsilon_{m}=2(m \neq 0)$, and where the $P_{n}^{m}$ are the associated Legendre polynomials. On inserting the above expression for $1 / R$, and utilizing orthogonality, eq (18) reduces to

$$
A^{p}=\frac{I \sin \beta}{2} \sum_{n=1}^{\infty} \frac{P_{n}^{1}(\cos \theta) P_{n}^{1}(\cos \beta)}{n(n+1)}\left\{\begin{array}{l}
(b / r)^{n+1} ; \quad r>b \\
(r / b)^{n} ; r<b,
\end{array}\right.
$$

which is the desired result.

\section{References}

[1] C. T. Tai, Hertzian dipole immersed in a dissipative medium, Cruft Lab. Report No. 21 (Harvard University 1947).

[2] J. R. Wait, Transient electromagnetic wave propagation in a conducting medium, Geophysics 16, 213 (1951) ; 18, 138 and 971 (1953); see also Ph. D. Thesis, University of Toronto (March 1951).

[3] R. K. Moore, Theory of radio communication between submerged submarines (Ph. D. Thesis, Cornell University, 1951).

[4] J. R. Wait, The magnetic dipole on the horizontally stratified earth, Can. J. Phys. 29, 577 (Nov. 1951).

[5] J. R. Wait, The magnetic dipole antenna immersed in a conducting medium, Proc. Inst. Radio Engrs. 40, 1244 (Oct. 1952).

[6] J. R. Wait and L. L. Campbell, Fields of an oscillating magnetic dipole immersed in a semi-infinite conducting medium, J. Geophys. Research 58, 167 (June 1953).

[7] R. H. Lien, Radiation from a horizontal dipole in a semi-infinite dissipative medium, J. Appl. Phys. 24, 1 (Jan. 1953) (see a discussion of this paper by J. R. Wait, J. Appl. Phys. 24, 958 (July 1953) in equation (6), V should be replaced by U).

[8] A. Banos and J. P. Wesley, The horizontal electric dipole in a conducting half-space, University of California, Marine Physical Lab., Pt. I, S10 Ref. 53-33, Sept. 1953, and Pt. II, S10 Ref. 54-31 (Aug. 1954).

[9] H. A. Wheeler, Fundamental limitations of a small V. L. F. antenna for submarines, Paper No. 14 (Symposium on V. L. F. Radio Waves, January 23-25, 1957, Boulder, Colo.).

[10] P. M. Morse and H. Feshbach, Methods of theoretical physics, vol. II, p. 1326 (MeGraw-Hill Book Co., Inc., New York, N. Y., 1953).

Boulder, Colo., February 15, 1957. 